\title{
A STUDY ON STRESS, DEPRESSION AND SUICIDAL IDEATION ACCORDING TO SEXUAL BEHAVIOURS AMONG KOREAN ADOLESCENTS
}

\author{
Eunmi Lee, Yujeong Kim \\ Department of Nursing, Research Institute for Basic Sciences, Hoseo University, Asan, Korea
}

\section{SUMMARY}

Objective: The purpose of this study was to examine stress, depression and suicidal ideation in Korean adolescents according to their sexual behaviours, such as experience of sexual intercourse, time of first sexual intercourse, and sexual intercourse after alcohol drinking.

Methods: This study was conducted as a secondary data analysis using the raw data of the 9th Korea Youth Risk Behaviour Web-based Survey (KYRBWS-IX) conducted in 2013. The survey sampled 75,149 secondary school students, and 72,435 of them were included in this analysis.

Results: Stress level, depression and suicidal ideation were significantly higher in adolescents with experience of sexual intercourse than in those without it. Adolescents with experience of sexual intercourse after drinking had a 1.415 times higher odds ratio depression than those without it. When the time of first sexual intercourse was delayed by one year, the odds of stress increased by 1.022 times and the odds of suicidal ideation increased by 1.014 times.

Conclusions: Adolescents with experience of sexual intercourse showed significantly higher stress, depression and suicidal ideation, the latter of which was particularly higher among low-grade female students that had experienced first sexual intercourse at a young age. Suicidal ideation was more common when first sexual intercourse was early. Adolescents with experience of sexual intercourse after drinking showed higher stress, depression and suicidal ideation than those without drinking.

Key words: adolescent, coitus, stress, depression, suicidal ideation

Address for correspondence: Yujeong Kim, Department of Nursing, Hoseo University, 20 Hoseo-ro, 79 beon-gil, Asan 31499, Korea. E-mail: yujeong@hoseo.edu

https://doi.org/10.21101/cejph.a4804

\section{INTRODUCTION}

Adolescence is an important and sensitive period characterized by emotional growth and various physical changes, and adolescents are very interested in the other sex and curious about sexual acts. In fact, it has been reported that $40 \%$ of Korean adolescents have dated (1), 71\% of those dating had experience of physical contact, and $18 \%$ experience of sexual intercourse (2). However, during such a sensitive period, sexual intercourse may cause physical and mental confusion and have negative impacts. In particular, sexual intercourse experienced during adolescence hinders normal physical growth, leads to the adoption of a passive attitude in social life, and induces various problem behaviours, such as smoking, drinking, running away, depression, and suicide (3-5). Female adolescents that experienced sexual intercourse without emotional sympathy or at a young age are more likely to have depression (4). Furthermore, sexual intercourse among adolescents is increasing and the age of sexual debut is reducing, and many adolescents receiving incorrect information on sexual intercourse and pregnancy are likely to suffer due to pregnancy or venereal disease $(6,7)$, and from psychological pains associated with deviation from social norms (8-10).

In Korea, where social attitudes to sex are conservative, the national percentage of adolescents with experience of sexual intercourse is lower than in other countries (11), but physical and psychological pains resulting from sexual intercourse may not be comparable with those determined previously in other countries. Due to its seriousness, adolescent sexual intercourse and sexual behaviour have been studied by many researchers, but few studies have been made on the relationship between adolescent mental health, such as stress, depression and suicidal ideation, and sexual behaviours based on national survey data.

Thus, the purpose of this study was to examine stress, depression and suicidal ideation among Korean adolescents according to their sexual behaviours, such as experience of sexual intercourse, time of first sexual intercourse, and sexual intercourse after drinking, and also to explore the seriousness of sex-related issues among Korean adolescents. The specific goals of the study were as follows: to assess correlation relations between general characteristics and experience of sexual intercourse; to identify control variables by analysing correlation relations between general characteristics and stress, depression and suicidal ideation; to model the effect of experience of sexual intercourse on stress, depression and suicidal ideation for each control variable, and subsequently to test the model; and to model how sexual behaviours affect stress, depression and suicidal ideation for each control variable and to test the model. 


\section{MATERIALS AND METHODS}

\section{Subjects}

This study was conducted as a secondary data analysis using the raw data of the 9th Korean Youth Risk Behaviour Web-based Survey (12) in order to analyse differences in adolescent stress, depression and suicidal ideation according to their sexual behaviours. Data were obtained from the 9th Korean Youth Health Risk Behaviour Online Survey published by the Korean Centres for Disease Control and Prevention. The 9th Korean Youth Health Risk Behaviour Online Survey was an anonymous self-administered online survey and was conducted from the 1st to the 30th of June 2013. In this survey, secondary school students from the 7th to 12th grades (as of April 2012) were stratified according to school type and area, where the sample school was first extracted and the class in the sample school was second. As a result, 75,149 students from 400 middle schools and 400 high schools were sampled, and 799 schools and 72,435 students participated in the survey (a participation rate of $96.4 \%$ ).

\section{Questionnaire and Definition of Variables}

\section{Sexual Behaviours}

Sexual behaviours were defined as experience of sexual intercourse, time of first sexual intercourse, and sexual intercourse after drinking. Experience of sexual intercourse was answered 'Yes' (1) or 'No' (0). The time of first sexual intercourse was determined by asking 'When did you first have sexual intercourse? (grade)' and the answer was treated as a continuous variable. Experience of sexual intercourse after drinking was surveyed by asking 'Have you ever had sexual intercourse after drinking?' and it was answered 'Yes' (1) or 'No' (0).

\section{Stress}

Stress was surveyed by asking 'How much stress do you usually feel?' and it was answered using a 5-point Likert scale, which ranged from 'Not at all' (1) to 'Very high' (5). It was measured as a quantitative variable and was then converted into 'No' (1-2 points) and 'Yes' (3-5 points) in this study.

\section{Depression}

Depression was surveyed by asking 'During the last 12 months, have you ever felt sadness or despair to the extent that you withdrew from the activities of daily living for over two weeks?', and it was answered 'Yes' (1) or 'No' (0).

\section{Suicidal Ideation}

Suicidal ideation was surveyed by asking 'During the last 12 months, have you ever had a serious suicidal idea?' and it was answered 'Yes' (1) or 'No' (0).

\section{Socio-demographic and Socioeconomic Status}

As the findings of previous studies and this study suggested that general characteristics, such as gender, grade, parents' education level, school achievement, economic status, and inhabitation type might confound results, they were used as control variables in the result analysis models. Fathers' and mothers' education levels were categorized as middle school graduate or lower, high school graduate, and college graduate or higher. School achievement and economic status were surveyed by asking 'How well have you performed at school during the last 12 months?' and 'How do you rate your family's economic status?'. Responses were categorized as very high, high, moderate, low, and very low grade. Inhabitation type was categorized as living with family or relatives or living alone.

\section{Statistical Analyses}

Considering the educational environment, all data were classified into middle school students (equivalent to US grades 7-9) and high school students (equivalent to US grades 10-12) before performing the analysis. Since the Korea Youth Risk Behaviour Web-based Survey was conducted with a complex sampling design using stratified three stage cluster sampling, the data were analysed with a complex sampling estimator using weights (such as proc surveyfreq and proc surveylogistic in SAS 9.2). To determine the control variables, a homogeneity test using the chi-square distribution was conducted to compare differences in stress, depression and suicidal ideation by general characteristics (gender, education level of parents, and living arrangement), and the logistic regression was performed to compare stress, depression and suicidal ideation by grade, school achievement and economic status. To examine the effects of sexual intercourse experience on stress, depression and suicidal ideation, logistic regression analysis was performed using general characteristics as control variables. To identify the influence of the main factors on the subjects with sexual intercourse experience, the above analysis was performed only on subjects with sexual intercourse experience with the time of first sexual intercourse and the experience of sexual intercourse after drinking set as independent variables.

\section{RESULTS}

\section{General Characteristic and Effects on Stress, Depres- sion and Suicidal Ideation}

Among 72,435 subjects, there were 3,867 (5.3\%) adolescents with and 68,568 (94.7\%) without sexual intercourse experience. Of the subjects who had sexual intercourse experience, 2,740 $(70.9 \%)$ were male students and $1,127(29.1 \%)$ were female students.

The analysis of stress, depression and suicidal ideation by general characteristics showed statistically significant differences in most variables (Table 1). A higher proportion of female students experienced stress, depression and suicidal ideation than male students. The proportion of high school students who have experienced stress and depression was higher than middle school students, but the opposite was true for suicidal ideation. In terms of the education level of parents, the lower the education level of the mother, the higher the rate of stress, depression and suicidal ideation in both middle and high school students. On the other hand, the lower the education level of the father, the higher the experience rate of stress and depression in middle school students, while the experience rate of depression and suicidal ideation was higher in high school students. In case of living arrangement, no 


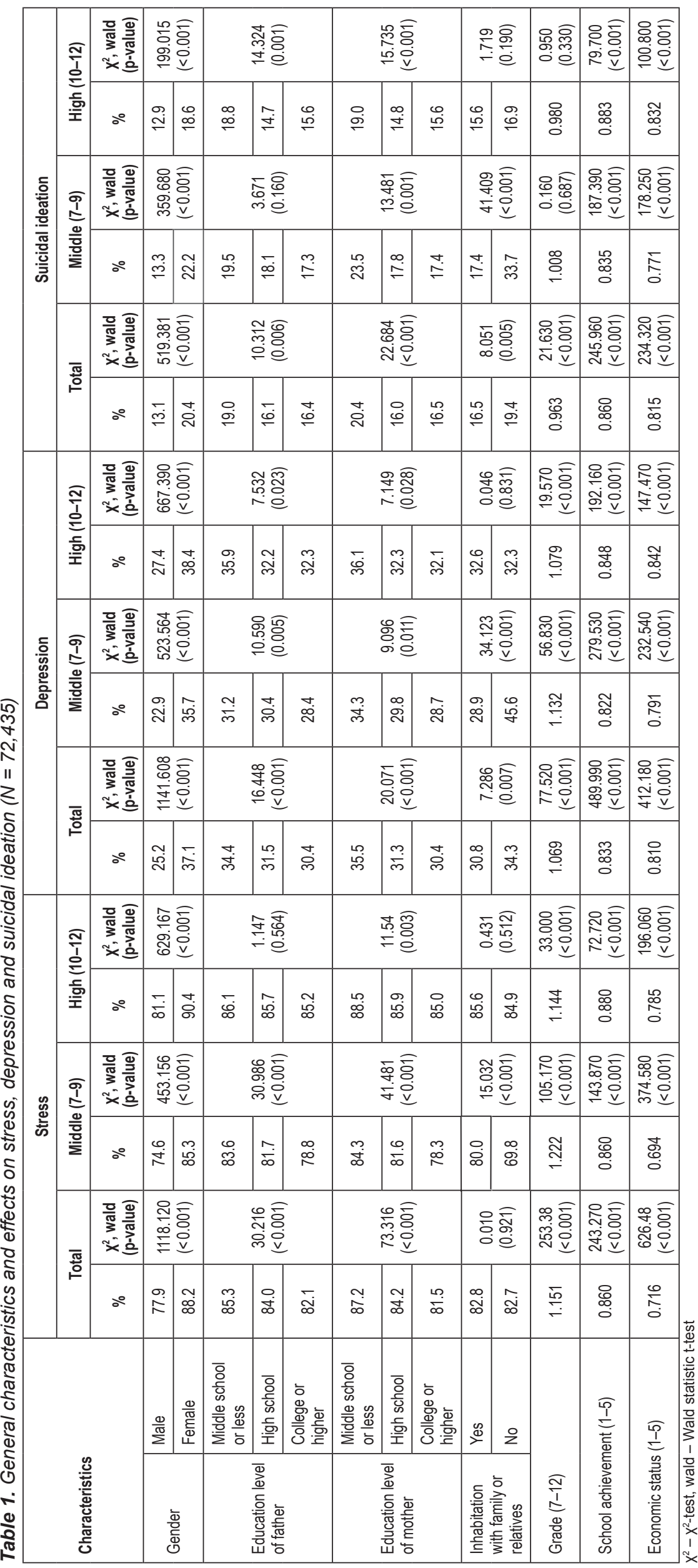




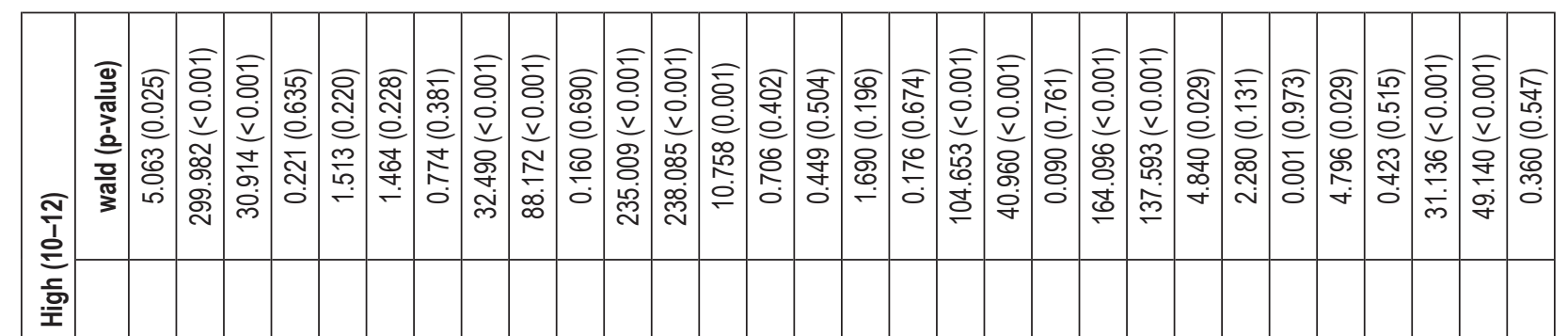

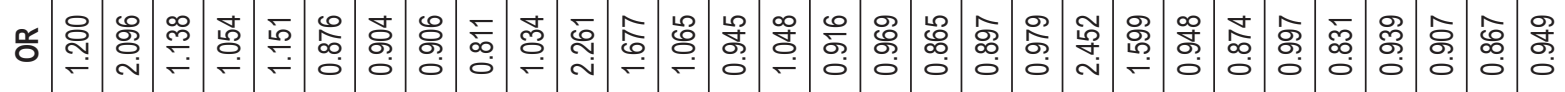

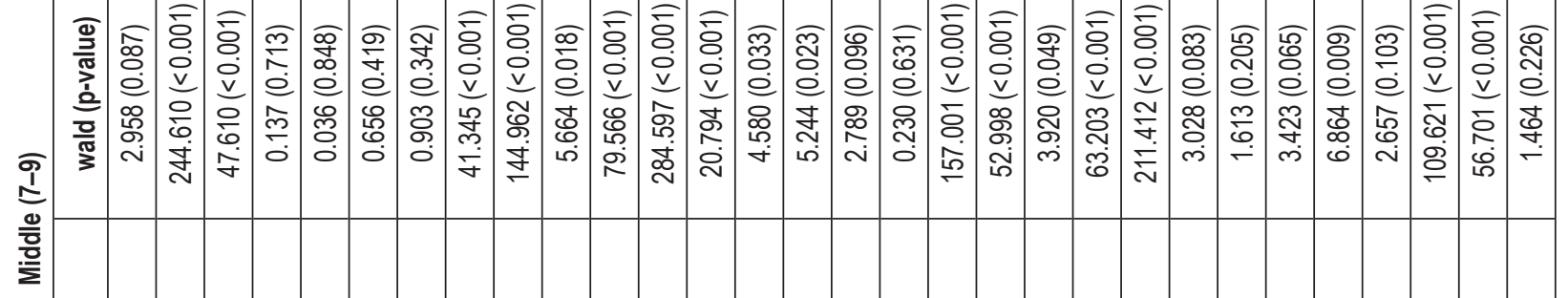

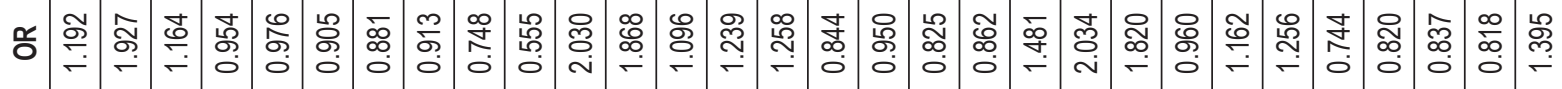

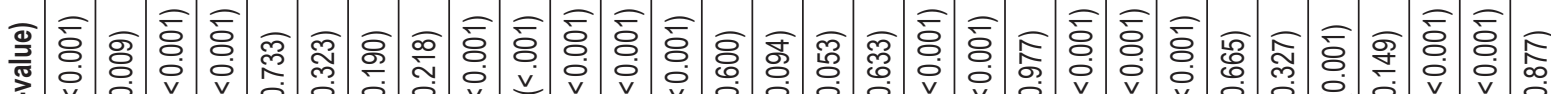

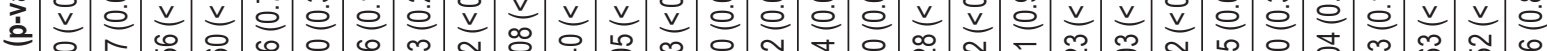

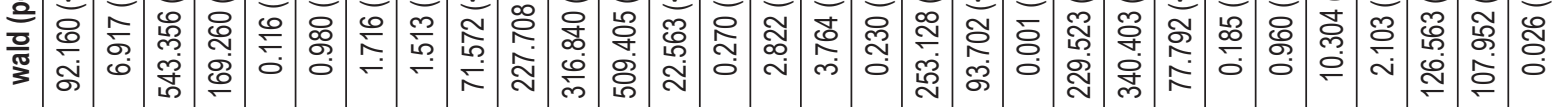

昰

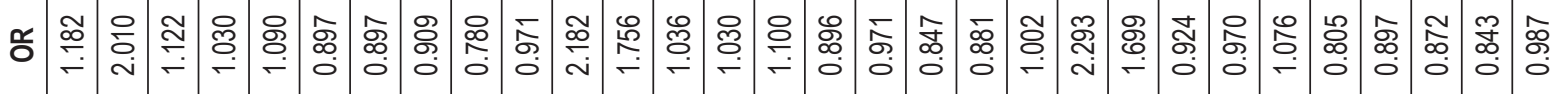

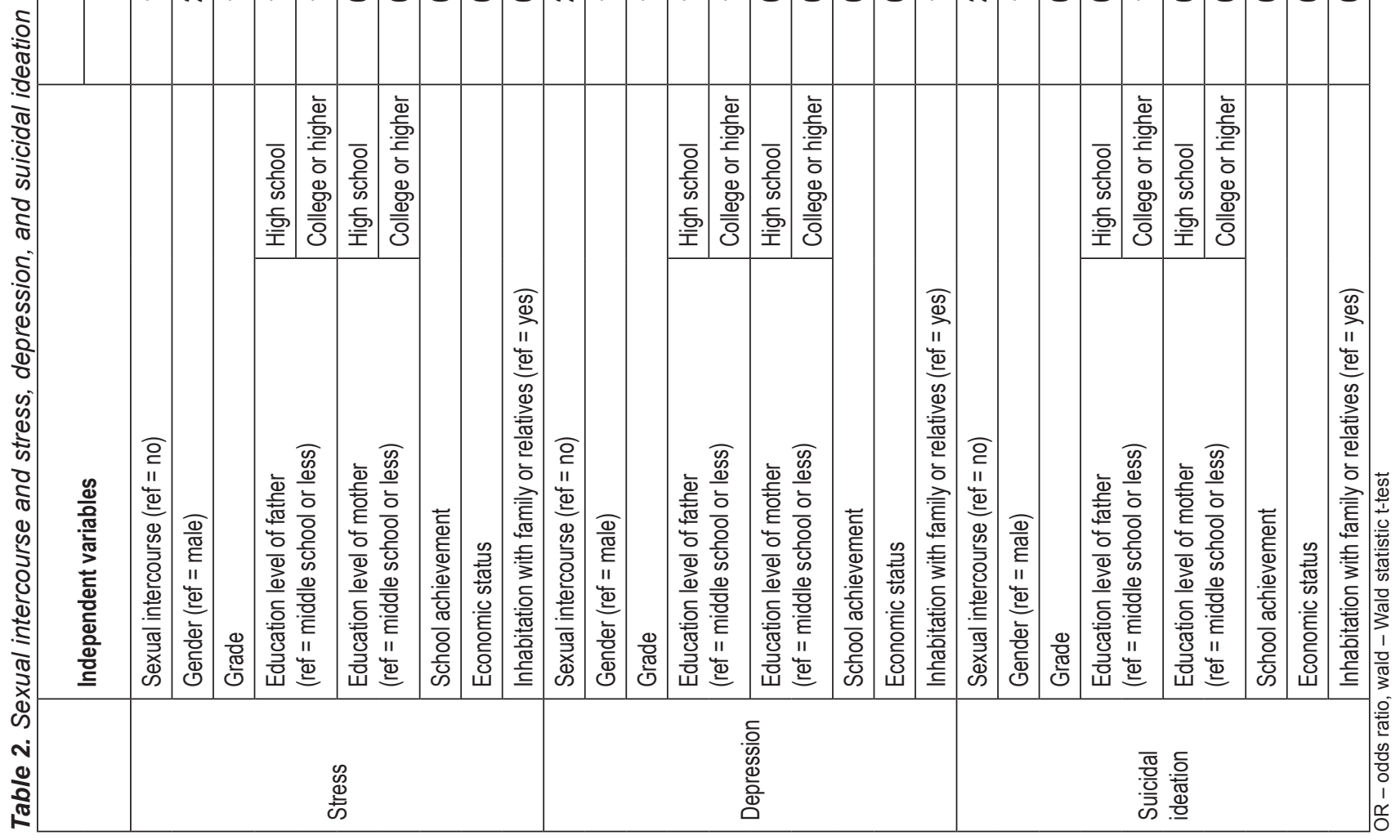




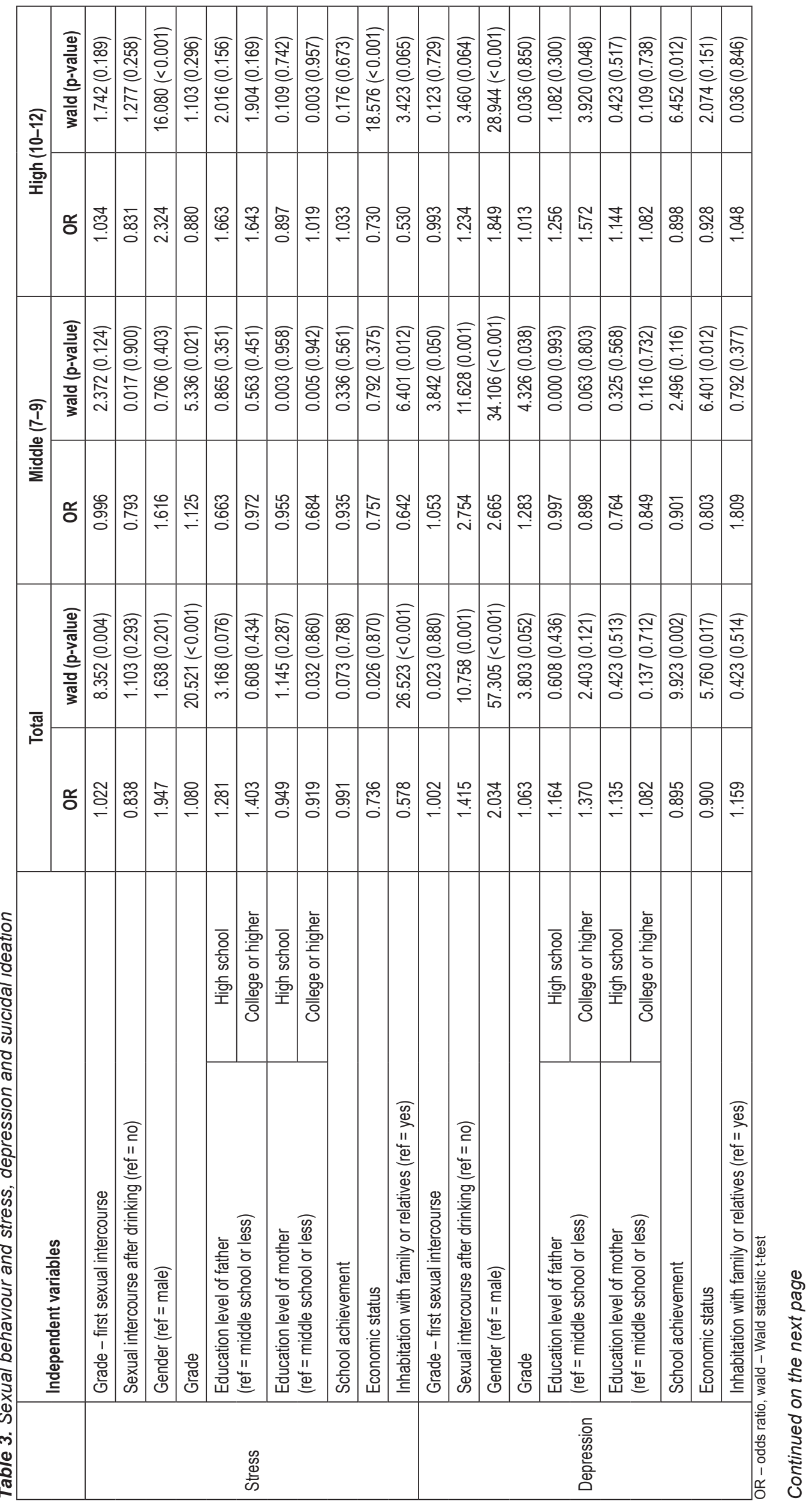




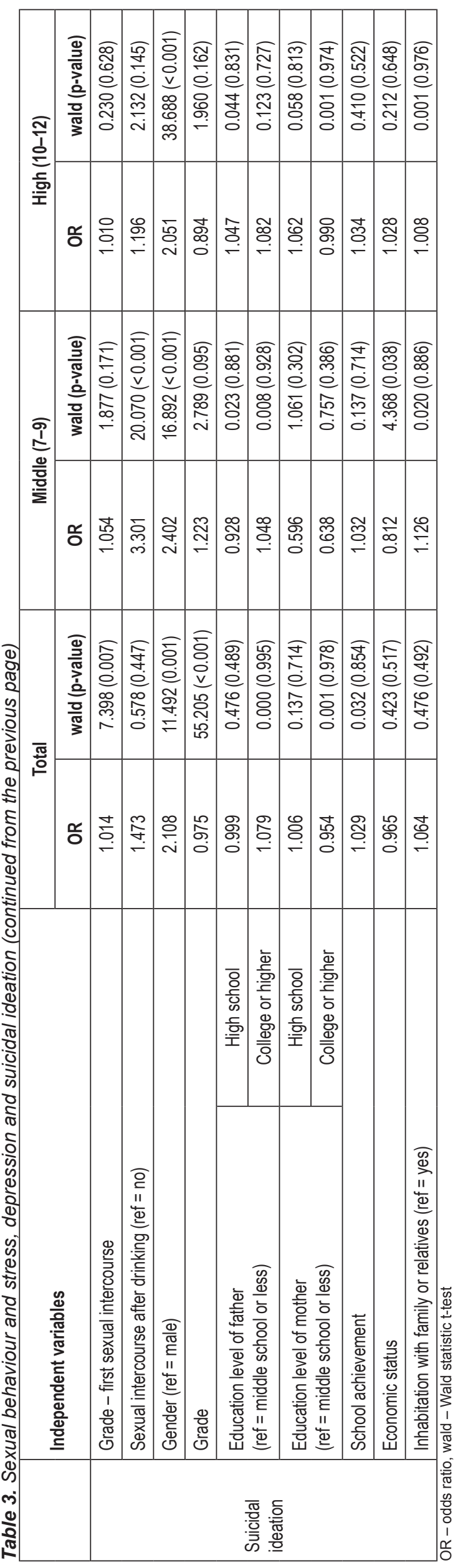

difference in stress was found regardless of whether the subjects live alone or with family or relatives. For middle school students, however, the experience rate of stress among adolescents living with family or relatives was higher than those living alone. For depression and suicidal ideation, the experience rate of depression was higher for students living with family or relatives than those living alone, but no difference was found in high school students.

In terms of grade, the higher the grade, the higher the experience rate of stress and depression, while the experience rate of suicidal ideation was lower. For both middle and high school students, the experience rate of stress and depression increased as they went up the grades, but no difference was found in the experience rate of suicidal ideation. It was found that the higher the school achievement and economic status, the lower the experience rate of stress, depression and suicidal ideation.

\section{Experience of Sexual Intercourse and Stress, Depres- sion and Suicidal Ideation}

Table 2 shows the results of the logistic regression analysis conducted to analyse the influence of stress, depression and suicidal ideation according to the experience of sexual intercourse with all general characteristics of the subjects (gender, grade, education level of parents, school achievement, economic status, and living arrangement) controlled.

In terms of the influence of stress, a statistically significant influence of sexual intercourse experience on stress was found. The odds of stress of adolescents with sexual intercourse experience were 1.182 times higher than that of their counterparts. A statistically significant difference was not found for middle school students. The odds of stress of high school students with sexual intercourse experience were, however, 1.200 times higher than that of their counterparts.

The influence of sexual intercourse experience on depression was statistically significant. The odds of depression of adolescents with sexual intercourse experience were 2.182 times higher than that of their counterparts. The influence of sexual intercourse experience on the odds of depression was found to be higher for high school students, and the odds of depression of adolescents with sexual intercourse experience for middle and high school students were 2.030 and 2.261 times higher, respectively, than those of their counterparts.

In terms of suicidal ideation, the influence of sexual intercourse experience on suicidal ideation was statistically significant. The odds of suicidal ideation of adolescents with sexual intercourse experience were 2.293 times higher than that of their counterparts. The influence of sexual intercourse experience on the odds of suicidal ideation was found to be higher for high school students, the odds of suicidal ideation of adolescents with sexual intercourse experience for middle and high school students were 2.034 and 2.452 times higher, respectively, than those of their counterparts.

\section{Sexual Behaviours and Stress, Depression and Sui- cidal Ideation}

To analyse the influence of stress, depression, and suicidal ideation according to sexual behaviours such as the time of first sexual intercourse and sexual intercourse after drinking, only the data of adolescents with sexual intercourse experience were analysed. 
The influence of the time of first sexual intercourse on stress was significant, and the odds of stress increased by 1.022 times when the time of first sexual intercourse was delayed by one year. When the data were classified into middle and high school students, however, the influence of the time of first sexual intercourse on stress was nonsignificant. The influence of sexual intercourse after drinking on stress was nonsignificant as well.

The influence of the time of first sexual intercourse on depression was nonsignificant for both middle and high school students. The influence of sexual intercourse after drinking on depression was significant for middle school students, and the odds of depression of adolescents with sexual intercourse after drinking were 2.754 times higher than that of their counterparts.

Even though the odds of suicidal ideation were 1.014 times higher when the time of first sexual intercourse was delayed by one year, the influence was nonsignificant when the data of middle and high school students were analysed separately. In terms of the influence of sexual intercourse after drinking on suicidal ideation, the odds of suicidal ideation of adolescents with sexual intercourse after drinking were 3.301 times higher than that of their counterparts, but no significant influence was found for high school students.

\section{DISCUSSION}

According to the results of the 2013 Korean Youth Health Risk Behaviour Online Survey (12), the average age of adolescents with experience of sexual intercourse was 12.8 years, which is 0.8 years younger than 13.6 years reported for the previous year, and it indicates that adolescents have first sexual intercourse in the 5 th or 6 th grade of elementary school on average. According to the results of this study, adolescents with experience of sexual intercourse showed higher levels of stress, depression and suicidal ideation, and suicidal ideation was particularly higher in low-grade female students with experience of first sexual intercourse at a young age. This finding is consistent with a report that adolescents with experience of sexual intercourse are more likely to have depression and to attempt suicide (5), and with a report that sexual experience had a higher negative impact on female adolescents (13). These findings suggest that sexual intercourse experienced by young female adolescents, who are physically and emotionally immature, induces more severe psychological effects.

In Korea, people are seeking more sexual freedom and satisfaction, yet among women, the perception of sex is still strongly conservative and attached to the notion of premarital chastity (14). Because adolescents are required to commit themselves to school studies, female students who have experienced early sexual intercourse are believed to suffer heavy psychological burdens, such as stress, depression and suicidal ideation and anxiety about unwanted pregnancy, venereal disease, and guilt. Importantly, the outcomes of this study are not considered comparable with those of previous studies in foreign countries where levels of sexual freedom are considerably different. Accordingly, the government needs to develop programmes and establish legal systems and education systems, including the obligatory allocation of hours for more substantial and satisfactory sex education from the elementary school level.

In the present study, adolescents with experience of sexual intercourse after drinking showed significantly higher depres- sion and suicidal ideation than those without it, even after correcting for general characteristics, such as gender, grade, school achievement, economic status, and inhabitation type. Drinking impairs judgment and the ability to control sexual desire, and as a result, increases the likelihood of impulsive sexual intercourse (15-18). In addition, intoxicated individuals are less sensitive to risk and often fail to use a contraceptive. Previous studies have reported that adolescent drinking is a major cause of accidents, dangerous sexual acts, venereal diseases, pregnancy, sexual abuse, and sex-related crimes (19-21). In Korea, adolescent drinking is not properly regulated despite the age limit imposed on alcohol purchase by the Juvenile Protection Act (12), and protective policies for regulating drinking-related harmful environments are not sufficient. Given this situation, it is time to reinforce national preventive interventions, campaigns, and to change perceptions regarding adolescent drinking. In addition, schools should intervene to change the attitudes and outcomes of alcohol drinking behaviours in adolescents by applying anti-alcohol campaign, promoting self-efficacy, stress management, counselling, and family or self-help group based prevention. Social and environmental efforts should be increased to ensure that adolescents receive regular care of their physical and mental health and are provided with preventive interventions to promote health and self-care.

When the analysis was carried out separately for middle school (equivalent to US grades 7-9) and high school students (equivalent to US grades 10-12), depression and suicidal ideation were significantly higher for middle school students who had sexual intercourse after drinking than those who had not, but the differences were nonsignificant for high school students. The reason may be that, due to the influence of media, high school students have expectations that sexual experiences can be easier and more enjoyable after drinking (22). Another reason may be that sexual experiences with a romantic partner along with drinking are not negative experiences that cause depression and suicidal ideation for older teenagers who are close to adulthood (23). On the other hand, early sexual experience has been reported to negatively influence sexual health in the long run because early sexual experience before the age of 16 years lets them have sexual experience under the influence of alcohol and drugs even after they become adults, and it increases the chances of contracting sexually transmitted diseases (24). Therefore, it is necessary for adolescents to protect their own health to reduce and prevent problems due to early sexual experience accompanied by drinking, and to promote health, including sexual health, not only through short sex education but also overall health education programmes including substance abuse, smoking and drinking.

This study confirms the association between adolescent sexual behaviour and mental health and presents a basis for preventing dangerous sexual behaviours. However, this is a cross-sectional survey, so the causal relationship between adolescent sexual behaviour and mental health could not be ascertained. In addition, the reverse causality may be possible. Thus, further research is needed on longitudinal research of addressing the psychological damage caused by adolescent sexual behaviour. Because the study was based on a secondary data analysis of data extracted from a self-administered questionnaire survey, participants' answers may not have been accurate. In addition, this study has a limitation in terms of not controlling for all demographic, physical, psychological, and environmental factors that have been identified 
as risk factors when analysing the relation of stress, depression and suicidal ideation with sexual behaviours. There is a need to investigate influencing factors and paths such as personal factors, school system factors, and social system factors to understand sexual behaviours and mental health in adolescents from various aspects.

\section{CONCLUSION}

In this study the effects of experience of sexual intercourse and sexual behaviours, such as time of first sexual intercourse and sexual intercourse after drinking, on stress, depression and suicidal ideation were investigated in Korean adolescents. It was found that those with a low parental education level, not living together with family or relatives, with a low school achievement level, with a poor economic status, those in higher grades and male students had higher rates of experience of sexual intercourse. Stress, depression and suicidal ideation were higher in those with experience of sexual intercourse than in those without it, and suicidal ideation was particularly higher in low-grade female students who had first sexual intercourse at a young age. The suicidal ideation rate was higher for those with an early sexual debut, and adolescents with experience of sexual intercourse after drinking showed higher stress, depression, and suicidal ideation than those without drinking alcohol.

The findings of this study provide useful information for developing and applying interventions to reduce the psychological damage caused by adolescent experience of sexual intercourse and for developing programmes designed to prevent dangerous sexual intercourse.

\section{Acknowledgement}

This study was conducted after obtaining approval from the Institutional Review Board of the researcher's university (1041231-150811-HR033-01).

\section{Conflict of Interests}

None declared

\section{REFERENCES}

1. Lee MH. 2013 Seoul youth sex culture research. Seoul: Aha Sexuality Education \& Counseling Center for Youth; 2013.

2. Kim JS, Cho SW. An analysis of current status of sexual behaviors and consciousness about sex of adolescents: on adolescents including elementary school students. J School Soc Work. 2010;19:61-87.

3. Kim DS, Kim HS. Early initiation of alcohol drinking, cigarette smoking, and sexual intercourse linked to suicidal ideation and attempts: Findings from the 2006 Korean youth risk behavior survey. Yonsei Med J. 2010;51(1):18-26.

4. Meier AM. Adolescent first sex and subsequent mental health. Am J Sociol. 2007;112(6):1811-47.
5. Johnson KA, Noyes LR, Rector R. Sexually active teenagers are more likely to be depressed and to attempt suicide [Internet]. Washington, DC: The Heritage Foundation; 2003 [cited 2014 April 10]. Available from: http://www.heritage.org/research/reports/2003/06/sexually-activeteenagers-are-more-likely-to-be-depressed.

6. Ryu EJ, Choi SY, Kim YH. Factors associated with female adolescents' sexual experience. J Kor Soc Matern Child Health. 2004;8(2):239-50.

7. Wellings K, Nanchahal K, Macdowall W, McManus S, Erens B, Mercer $\mathrm{CH}$, et al. Sexual behavior in Britain: early heterosexual experience. Lancet. 2001;358(9296):1843-50.

8. Cyranowski JM, Frank E, Young E, Shear MK. Adolescent onset of the gender difference in lifetime rates of major depression: a theoretical model. Arch General Psych. 2000;57(1):21-7.

9. Guggino JM, Ponzetti JJ Jr. Gender differences in affective reactions to first coitus. J Adolesc. 1997;20(2):189-200.

10. Rodriguez C Jr, Moore NB. Perceptions of pregnant/parenting teens: reframing issues for an integrated approach to pregnancy problems. Adolescence. 1995;30(119):685-706.

11. Cavazos-Rehg PA, Krauss MJ, Spitznagel EL, Schootman M, Bucholz KK, Peipert JF, et al. Age of sexual debut among US adolescents. Contraception. 2009;80(2):158-62.

12. Ministry of Education; Ministry of Health and Welfare; Korea Centers for Disease Control and Prevention. The Ninth Korea Youth Risk Behavior Web-based Survey. Seoul: Korea Centers for Disease Control and Prevention; 2013.

13. Hallfors DD, Waller, MW, Ford CA, Halpern CT, Brodish PH, Iritani B. Adolescent depression and suicide risk: association with sex and drug behavior. Am J Prev Med. 2004;27(3):224-31.

14. Hur TK, Han M, Kim YS. Gender differences of risk-taking decisions in sexual behaviors: condom use and theory of planned behavior. Kor $\mathrm{J}$ Psych. 2004;9(3):69-87.

15. Gwon SH, Lee CY. Factors that influence sexual intercourse among middle school students: using data from the 8th (2012) Korea youth risk behavior web-based survey. J Kor Acad Nurs. 2015;45(1):76-83.

16. Nkansah-Amankra S, Diedhiou A, Agbanu HL, Harrod C, Dhawan A. Correlates of sexual risk behaviors among high school students in Colorado: analysis and implications for school-based HIV/AIDS programs. Matern Child Health J. 2011;15(6):730-41.

17. Rew L, Carver T, Li CC. Early and risky sexual behavior in a sample of rural adolescents. Iss Comprehens Pediatr Nur. 2011;34(4):189-204.

18. Santelli JS, Kaiser J, Hirsch L, Radosh A, Simkin L, Middlestadt S. Initiation of sexual intercourse among middle school adolescents: the influence of psychosocial factors. J Adolesc Health. 2004;34(3):200-8.

19. Boden JM, Fergusson DM. The short and long-term consequences of adolescent alcohol use. In: Saunders JB, Rey JM, editors. Young People and Alcohol: Impact, Policy, Prevention, Treatment. Chichester: WileyBlackwell; 2011.

20. Champion HL, Foley KL, DuRant RH, Hensberry R, Altman D, Wolfson M. Adolescent sexual victimization, use of alcohol and other substances, and other health risk behaviors. J Adolesc Health. 2004;35(4):321-8.

21. Foshee VA, Benefield TS, Ennett ST, Bauman KE, Suchindran C. Longitudinal predictors of serious physical and sexual dating violence victimization during adolescence. Prev Med. 2004;39(5):1007-16.

22. Hartley JE, Wight D, Hunt K. Presuming the influence of the media: teenagers' constructions of gender identity through sexual/romantic relationships and alcohol consumption. Soc Health Illness. 2014;36(5):772-86.

23. Franz A, Worrell M, Vögele C. Discourses of sexual relationships in a sample of German and British young people: a Q methodological study. Cult Health Sexual. 2016;18(4):391-404.

24. Sandfort TG, Orr M, Hirsch JS, Santelli J. Long-term health correlates of timing of sexual debut: results from a national US study. Am J Public Health. 2008;98(1):155-61.

Received April 18, 2016 Accepted in revised form September 11, 2018 